\title{
miRNASNP: a database of miRNA related SNPs and their effects on miRNA function
}

\author{
Jing Gong, Yin Tong, Hong-Mei Zhang, An-Yuan Guo* \\ From Eighth International Society for Computational Biology (ISCB) Student Council Symposium 2012 \\ Long Beach, CA, USA. 13-14 July 2012
}

\section{Background}

MicroRNAs (miRNAs) are a family of endogenous small non-coding RNAs involved in various developmental and physiological processes by negatively regulating gene expression. Single nucleotide polymorphisms (SNPs) are important variations for the diversity among individuals, as well as leading to phenotypes, traits, and diseases. What will happen when SNPs meet miRNAs? To date, a number of studies have demonstrated that SNPs in target sites or miRNA genes were associated with diseases [1-3]. Our aim is to characterize these functional miRNA related SNPs comprehensively.

\section{Results}

Through mapping SNPs onto miRNAs, we totally identified 757 SNPs (including indel polymorphisms) in 440 human pre-miRNAs, 218 SNPs in pre-miRNAs of other 8 species and thousands of SNPs in pre-miRNAs flanking regions. Of them, 50 SNPs are in the seed regions of 41 human miRNAs. Two different methods (TargetScan and miRanda) were used to predict the target sites for the wild type miRNAs and SNP-miRNAs. By comparing the targets of wild type miRNA with that of SNP-miRNA, we obtained the potential miRNA targets loss and gain. We found about half of the targets will be changed by SNP in miRNA seed region. In addition, we experimentally confirmed seven loss of-function SNPs and one gain-of-function SNP by luciferase report assay.

We also mapped SNPs onto gene 3' untranslated region (3'UTR) and identified tens of thousands of SNPs in 3'UTRs which would affect the miRNA/gene interaction, either destroy a real target or create an illegal target. Among these miRNA related SNPs, we think the SNPs

\footnotetext{
* Correspondence: guoay@mail.hust.edu.cn

Hubei Bioinformatics \& Molecular Imaging Key Laboratory, Department of Biomedical Engineering, College of Life Science and Technology, Huazhong University of Science and Technology, Wuhan, 430074, China
}

with relatively high minor allele frequencies (MAF), high MAF difference between populations and undergoing positive selection pressure will be important candidates for population phonotype research and complex trait studies.

All useful data were compiled into miRNASNP, a user-friendly free online database (http://www.bioguo. org/miRNASNP/).

\section{Conclusions}

We have systematically identified and analyzed the human polymorphisms in miRNAs and miRNA target sites and analyzed their potential influences on target binding. Our database will be a useful resource for studying miRNA function, identifying disease-associated miRNAs, and further personalized medicine.

Published: 14 December 2012

\section{References}

1. Jazdzewski K, Murray EL, Franssila K, Jarzab B, Schoenberg DR, de la Chapelle A: Common SNP in pre-miR-146a decreases mature miR expression and predisposes to papillary thyroid carcinoma. Proceedings of the National Academy of Sciences of the United States of America 2008, 105(20):7269-7274

2. Sethupathy P, Borel C, Gagnebin M, Grant GR, Deutsch S, Elton TS, Hatzigeorgiou AG, Antonarakis SE: Human microRNA-155 on chromosome 21 differentially interacts with its polymorphic target in the AGTR1 3' untranslated region: a mechanism for functional single-nucleotide polymorphisms related to phenotypes. American journal of human genetics 2007, 81(2):405-413.

3. Sun $Q$, Gu H, Zeng Y, Xia Y, Wang Y, Jing Y, Yang L, Wang B: Hsa-mir-27a genetic variant contributes to gastric cancer susceptibility through affecting miR-27a and target gene expression. Cancer science 2010, 101(10):2241-2247.

doi:10.1186/1471-2105-13-S18-A2

Cite this article as: Gong et al.: miRNASNP: a database of miRNA related SNPs and their effects on miRNA function. BMC Bioinformatics 2012 13(Suppl 18):A2.
(C) 2012 Gong et al; licensee BioMed Central Ltd. This is an Open Access article distributed under the terms of the Creative Commons Attribution License (http://creativecommons.org/licenses/by/2.0), which permits unrestricted use, distribution, and reproduction in any medium, provided the original work is properly cited. 